\title{
Competitividad de la acuicultura española: Modelos explicativos
}

\author{
Cruz González, María Montserrat* \\ Guisado Tato, Manuel** \\ Sánchez Sellero, Francisco Javier***
}

\begin{abstract}
Resumen
El objetivo de este artículo es la determinación de modelos explicativos de la competitividad del sector acuícola en España, contrastando la incidencia en dichos modelos de factores endógenos contenidos en la teoría de recursos y capacidades. Tras la preceptiva recensión teórica, la metodología empleada consiste en el contraste y verificación de dos hipótesis sobre la aplicabilidad de dicha teoría respecto a una investigación empírica que combina resultados de una encuesta y ratios extraídos de la base de datos SABI de 99 empresas acuícolas españolas (mediante los programa SPSS y AMOS). Los resultados obtenidos (modelos factorial y path de ecuaciones estucturales) refieren la incidencia diferencial o prevalencia de ciertas variables sobre otras contenidas en la teoría, para el caso de la competividad de la acuicultura española. A la luz de la reespecificación de los modelos propuestos, podemos concluir que la competitividad de la acuicultura española se relaciona con la generación de valor, la productividad, el tamaño, la innovación y el grado de especialización.
\end{abstract}

Palabras clave: Competitividad, acuicultura, recursos, capacidades.

Recibido: 10-05-10. Aceptado: 20-09-10

* Doctora en Administración y Dirección de Empresas, Profesora Asociada, pertenece al Departamento de Organización de Empresas y Marketing, en la Facultad de Ciencias Económicas y Empresariales de la Universidad de Vigo. E-mail: mcruz@uvigo.es. Autora de contacto.

** Doctor en Ciencias Empresariales, Catedrático Universidad, pertenece al Departamento de Organización de Empresas y Marketing, en la Facultad de Ciencias Económicas y Empresariales de la Universidad de Vigo. E-mail: mguisado@uvigo.es.

*** Doctor en Administración y Dirección de Empresasas, Profesor Contratado Doctor, pertenece al Departamento de Organización de Empresas y Marketing, en la Facultad de Ciencias Económicas y Empresariales de la Universidad de Vigo. E-mail: javiss@uvigo.es. 
Competitividad de la acuicultura española: Modelos explicativos

Cruz González, María M.; Guisado Tato, Manuel y Sánchez Sellero, Francisco J.

\title{
Competitiveness in Spanish Aquaculture: Explanatory Models
}

\begin{abstract}
The aim of this paper is to determine explanatory models for the competitiveness of Spanish aquaculture, contrasting the effect of endogenous factors on the theory of resources and capacities in the above-mentioned models. After the obligatory theoretical review, the methodology consists of contrasting and verifying two hypotheses about the applicability of the above-mentioned theory to empirical research that combines results from a survey and ratios extracted from the SABI database of 99 Spanish aquaculture companies (using the SPSS and AMOS programs). The results obtained (factorial and path models of structural equations) indicate the differential incidence or prevalence of certain variables over others contained in the theory, for the case of competitiveness in Spanish aquaculture. From the re-specification of the proposed models, we can conclude that the competitiveness of Spanish aquaculture relates to the generation of value, productivity, size, innovation and specialization.
\end{abstract}

Key words: Competitiveness, aquaculture, resources, capabilities.

\section{Introducción}

En este artículo comenzamos determinando el marco teórico explicativo de la competitividad empresarial, a partir de las aportaciones de Wernerfelt, 1984; dicho autor precisó la especial significación que tienen los recursos y capacidades de una empresa como determinantes y depositarios del origen primario de cualquier tipo de ventaja competitiva accesible a las empresas. Aunque tuvo sus precursores, es este autor quien pone de relieve la existencia de un conjunto de factores endógenos, necesarios para la definición de estrategias competitivas, en mercados imperfectos.

Nuestro trabajo lleva a cabo un análisis minucioso de la teoría de recursos y capacidades y su incidencia sobre la competitividad empresarial, en particular contrastamos su aplicabilidad sobre el sector de la acuicultura en España.

Respecto a los fundamentos teóricos de la investigación proponemos el se- guimiento de tres fases o etapas conducentes al establecimiento de competencias distintivas básicas: la identificación, la clasificación y la valoración, tanto de recursos como de capacidades. Conceptualizamos diferencialmente ambos términos, evaluamos su trascendencia individual o colectiva, evidenciamos su etiología diversa y proponemos criterios para la valoración de ambas rúbricas. En concreto distinguimos entre recursos tangibles, intangibles y humanos (Guerras y Navas, 2008); al tiempo que la clasificación de competencias propuesta diferencia entre estratégicas, tecnológicas, organizativas y personales.

Una vez propuesto el marco teóricos de la investigación, buscamos su contraste para un sector de actividad en crecimiento estructural en los últimos años a nivel mundial, el sector elegido es la acuicultura y el marco territorial, España. Nuestro trabajo de campo trata de validar la incidencia de factores endógenos (recursos y 
capacidades) sobre la competitividad de la acuicultura española, a través de dos modelos de ecuaciones estructurales o de estructuras de covarianzas. Así asociamos la teoría de recursos y capacidades a la generación de valor, la productividad, la innovación o el grado de especialización de actividades, entre otros, como variables explicativas de la competitividad de la acuicultura española.

\section{Fundamentos teóricos: La teoría de recursos y capacidades}

La Teoría de Recursos y Capacidades sienta sus bases en la heterogeneidad de recursos entre empresas, así como en la imperfecta movilidad de los mismos; rasgos prioritarios que explicarán el porqué de las diferentes rentabilidades que obtienen las empresas con características, tanto organizativas como administrativas, similares; en nuestro caso el análsis lo aplicamos al sector de la acuicultura. Este enfoque parte de los factores internos que la empresa desarrolla y controla; para explicitar, en función de ellos, la emergencia de ventajas competitivas coadyuvantes a los objetivos y estrategias propuestos, sin que en esta precisa instancia interese, en particular, la protección de tales recursos o capacidades frente a la competencia (Wernerfelt, 1984; Penrose, 1995). Esta teoría define una estrategia que explotará los principales recursos y capacidades que posee la empresa, determinando la forma o manera eficiente de emplearlos conjuntamente y obtener los máximos beneficios posibles (Grant, 1996). La empresa se definirá como un conjunto de tecnologías y habilidades que se generan y amplían en el tiempo (Guerras y Navas, 2008); esto es, una combinación de recursos y capacidades heterogéneos que le proporcionan identidad a la organización empresarial, aún cuando el entorno varíe, puesto que son mucho más importantes las acciones internas que las externas.

Para poder establecer las competencias distintivas básicas, sobre las cuales podremos sentar las bases para la consecución y el desarrollo de las ventajas competitivas con las que cuentan las empresas acuícolas, es necesario identificar, clasificar y valorar correctamente los recursos y capacidades con los que cuentan, según su potencialidad de generación de beneficios (Figura 1).

a) Identificación: es importante considerar dos niveles básicos de análisis establecidos por Navas, Guerras y Grant. Por un lado, el carácter individual que presentan los recursos, que son controlados por la propia empresa y que se encuentran a disposición de la misma; y por otro lado, el carácter colectivo de las capacidades, consideradas como competencias o habilidades colectivas (Guerras y Navas, 2008) o el resultado de la utilización conjunta e interrelacionada de recursos (Grant, 1996).

b) Clasificación: al analizar esta función debemos precisar el límite que nos permitirá diferenciar los recursos de las capacidades, así como las correspondientes subdivisiones referentes a los tipos de recursos y tipos de capacidades que potencialmente puedan ser accesibles para las empresas.

b.1) Recursos; entendemos como tales todos aquellos bienes o factores que la empresa controla, su clasificación se 
Competitividad de la acuicultura española: Modelos explicativos

Cruz González, María M.; Guisado Tato, Manuel y Sánchez Sellero, Francisco J.

Figura 1. Esquema de análisis de los recursos y capacidades empresariales.
IDENTIFICACIÓN $\begin{cases}\hline \text { INDIVIDUAL } & \\ \text { COLECTIVO } & \text { RECURSOS } \\ \hline \text { CAPACIDADES }\end{cases}$

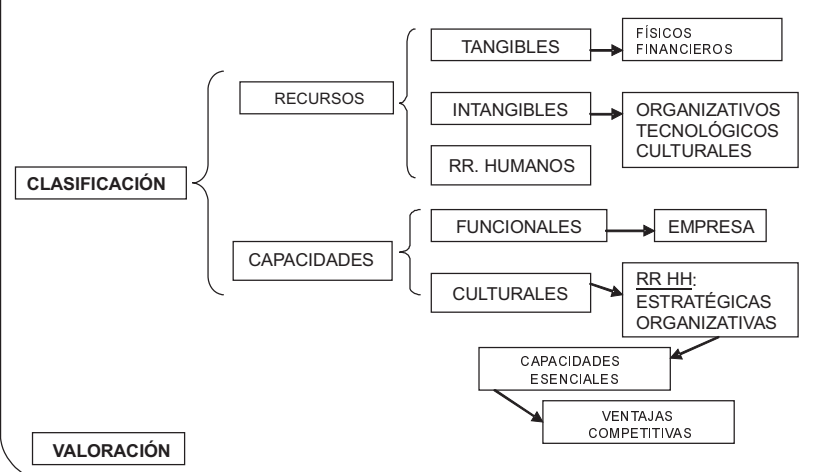
Fuente: Elaboración propia.
convierte en una tarea compleja, de he- cho existen diferentes enfoques sobre el tema. Penrose, 1995, es precursora en el análisis de los recursos, diferenciando entre recursos tangibles y recursos de naturaleza humana, siendo para esta au- tora una combinación de ambos la que permite el crecimiento de la empresa y no los factores externos a la misma, de he- cho es constatable la importancia de los recursos humanos en el sector acuícola (Polaco et al., 2000; González Laxe, 2001).
En este trabajo hemos optado por la división realizada por Navas, en recursos tangibles, intangibles y humanos. No deja- remos de lado la evolución tecnológica de
las empresas en el sector objeto de estu- dio, ya que gran parte la competitividad ra- dica en la combinación de recursos tangi- bles, intangibles y humanos. Para comple- tar la definición y caracterización genérica de los recursos, podemos afirmar que:
- Los recursos tangibles pueden presentarse bajo naturalezas tanto físicas como financieras. Su compor- tamiento permite observar el deno- minado efecto complementario (Ita- mi, 1987), cuyo objetivo es combinar elementos de la empresa con la fina- lidad de que se utilicen a plena capa- cidad $^{1}$. El único problema real de es- tos recursos se centra en su valora- ción contable.

1 La plena utilización de la capacidad de un recurso, se verá en casos como el de la utilización de un mismo activo físico para servir a dos mercados, utilizar dos medios de producción con igual intensidad a lo largo de un ciclo o utilizar recursos físicos en un mercado si hay fluctuaciones en otro. 
- Los recursos intangibles pueden ser de carácter tecnológico y organizativo, son recursos controlables por la empresa en la mayoría de los casos, y como ocurre con los recursos tangibles, son susceptibles de valoración a nivel empresarial mediante diferentes herramientas contables. Son recursos que se pueden obtener bien de forma exógena o bien de modo endógeno, dependiendo de si implican o no relaciones de la empresa con su entorno específico y genérico (Barney, 1991; Sveiby, 1997a y 1997b).

- Los recursos humanos están dotados de una característica especial, poseen personalidad propia, por lo que son muy distintos entre sí, de hecho inciden tanto positiva como negativamente en la empresa, en la medida en que son generadores de ventajas y desventajas para alcanzar los objetivos empresariales y mejorar la competitividad (Chiavenato, 2002). Son recursos que generan ventajas competitivas a través de sus conocimientos y de sus relaciones, y poseen competencias específicas resultado de la experiencia, el entrenamiento, la motivación, la capacidad de adaptación, la habilidad de razonamiento, la decisión, la lealtad a la empresa...

Son recursos cuya acumulación, al igual que en gran parte de los intangibles anteriores, se produce lenta- mente, de ahí que su coste pueda ser elevado; si bien su utilidad a nivel estratégico es muy grande, actuando como facilitadores de sinergias ${ }^{2}$ en la utilización simultánea de múltiples recursos, compartiendo tareas en distintas áreas de la empresa (Itami, 1987). Estos recursos, por tanto, no se deterioran con el uso, son combinables con el fin de alcanzar un mayor grado de conocimiento y se pueden valorar mediante su poder cognitivo, a través de magnitudes asociadas al capital humano y relacional (Barney, 1991; Sveiby, 1997).

Particularizando nuestra atención en los recursos intangibles, evidenciamos como se han clasificado en base a una serie de características que posibilitaran su integración diferencial, dada la complejidad que incorpora su análisis específico. A la hora de clasificarlos, uno de las posibilidades consiste en referir su importancia en la empresa, ya que no tienen el mismo valor, o no son de la misma naturaleza.

A lo largo de estos últimos años se han propuesto muchas más clasificaciones de los activos intangibles, demostrándose con ello la preocupación que éstos suscitan en la valoración de la empresa y en su estrategia. Se puede comprobar que en las diversas clasificaciones, los recursos humanos y sus capacidades aparecen como punto común, ya que enlazan con la posibilidad de obtener infor-

2 El efecto sinérgico es superior al complementario, si hablamos a nivel estratégico, aunque también es más difícil de conseguir. La adición de ambos efectos, nos lleva a definir el efecto cartera, que establece cómo utilizar y acumular los recursos con eficiencia y eficacia (Itami, 1987). 
Competitividad de la acuicultura española: Modelos explicativos

Cruz González, María M.; Guisado Tato, Manuel y Sánchez Sellero, Francisco J.

mación y transformarla en conocimiento. También es de destacar que, dentro de los intangibles, se le da una gran importancia al capital relacional, es decir, al conocimiento derivado de las vinculaciones entre diferentes puestos, actividades y funciones dentro de la empresa. En cierto modo, podemos resumir las aportaciones sobre la clasificación de los intangibles de los distintos organismos (públicos, privados y autores en general), en cuatro grandes grupos: aquéllos que se refieren a las personas y sus conocimientos, los organizacionales, los factores de carácter tecnológico y la reputación (Fernández Sánchez et al., 1999).

b.2) Capacidades: son también referidas como habilidades o competencias organizativas y permiten realizar de forma correcta cualquier actividad concreta dentro de la organización, a partir de la combinación y coordinación de los recursos individuales de los que dispone cada empresa. Tanto Selznick (1957) como Ansoff (1965), definieron las capacidades distintivas, el primero se refiere con ellas a "aquellas actividades que mejor hace la empresa", mientras que el segundo las definió como "el fundamento de las estrategias de crecimiento". En 1990, Hamel y Prahalad, pasan a denominarlas capacidades básicas, al distinguir entre aquellas capacidades inherentes a la empresa, de aquellas otras que solamente son periféricas.
Las capacidades más relevantes son aquéllas que surgen de la integración de otras ${ }^{3}$; así siguiendo las teorías seminales que Ansoff enunció en 1965, con las aportaciones y ampliaciones posteriores, consideramos su vinculación con los recursos humanos y las dividimos en cuatro tipos de capacidades:

- Las capacidades estratégicas son el resultado del "pensamiento o propósito estratégico que da origen a una acción constructiva y generativa"; también consideradas de este modo por Morcillo, en 1997. Podemos decir que son la conjunción de dos aspectos: por un lado, la misión y la cultura de la organización; y por otro, las características estructurales y funcionales de la empresa, derivadas de la actuación de los recursos humanos.

- Las capacidades tecnológicas, se establecen en función de las habilidades que poseen las empresas, a la hora de dominar una tecnología, para elaborar un bien o un servicio. Por tanto, se refieren a la capacidad de interrelacionar la visión, los recursos y las capacidades, tanto personales como técnicas, que poseen.

- Las capacidades organizativas parten de una correlación interna entre lo que sería la parte física de la empresa (infraestructuras, procesos, sistemas) y la cultura empresarial (estilo de dirección, formas de gobierno), es decir,

3 Los conceptos de competencia y capacidad son utilizados indistintamente (Hamel y Prahalad, 1995), ya que ambos implican la necesidad de su identificación previa o "a priori" en las empresas, de forma que debemos obtener ventajas competitivas tras el hallazgo originario de aquéllas: capacidades o competencias esenciales. 
las capacidades estratégicas. Sin embargo, es necesario que se cumpla el principio de homeóstasis, es preciso que la empresa se adapte a las variaciones del entorno y que acepte sus exigencias.

- Las capacidades personales, se derivan del éxito en la ejecución correcta de las tareas que implica cada puesto de trabajo y dependen de una serie de cualidades latentes y específicas de cada persona (Boyatzis, 1982). En base a cuanto antecede, pueden denominarse también competencias, esto es, conocimientos, experiencias y habilidades que permiten a las empresas diferenciarse (Loasby, 1999). Muchas veces, estas características personales se consiguen por la experiencia, en otros casos gracias al aprendizaje formativo, de hecho, los conocimientos forman parte esencial de las capacidades de una empresa (Richardson, 1977). En todo caso, conducen a la consecución de objetivos para la empresa y al desarrollo de iniciativas profesionales para los individuos.

En definitiva, el conjunto formado por las cuatro capacidades distintivas básicas: estratégicas, tecnológicas, personales y organizativas, generará las denominadas capacidades esenciales ${ }^{4}$. Tales capacidades afectarán a los comportamientos, tanto de las personas como de las empresas, y pueden transformarse entonces, en ventajas para la organiza- ción. Aunque el hecho de competir en capacidades no es nuevo en absoluto, la importancia del mismo radica en la profundización del concepto per se, que implica diferenciar entre competencias o capacidades "esenciales" y "no esenciales" (Hamel y Prahalad, 1995), dado que únicamente las primeras contribuirán a la consecución de ventajas competitivas. Así las capacidades esenciales han de cumplir tres condiciones: deben ayudar a aumentar el valor que perciben los clientes de los bienes y servicios ofrecidos por la empresa; a nivel competitivo, han de referir una capacidad única, diferencial o exclusiva, que no debe confundirse con una capacidad necesaria y finalmente; deben ser extensibles, o dicho de otro modo, deben posibilitar su aplicación y derivación en nuevas unidades estratégicas de negocio (Itami y Roehlt, 1987; Hamel y Prahalad, 1995).

c) Valoración: La implicación estratégica de los recursos y capacidades internos de la empresa, nos lleva a la necesidad de evaluarlos y valorarlos, respecto a su posible rentabilización futura. La definición de la estrategia actual nos debe permitir que planifiquemos hacia el futuro y podamos emplearlos en la estrategia futura, para que realmente puedan considerarse como recursos y capacidades distintivos o estratégicos (Guerras y Navas, 2008).

En una primera aproximación, Barney, 1997, expone el esquema VRIO, -valiosos, raros, inimitables y organiza-

$4 \quad$ La posibilidad de alcanzar una misma capacidad es siempre muy difícil, dado que suele manifestarse una coordinación de varios factores que limitan esta apropiación por parte de los rivales. 
Competitividad de la acuicultura española: Modelos explicativos

Cruz González, María M.; Guisado Tato, Manuel y Sánchez Sellero, Francisco J.

dos-, es decir, los recursos permitirán a la empresa responder a las amenazas y aprovechar las oportunidades del entorno, generar ventajas frente a competidores, fomentar la diferenciación y su explotación organizada, en la medida en que cumplan con las características citadas. Otra aproximación define hasta ocho criterios que nos permiten realizar una evaluación adecuada (Amit y Schoemaker, 1993): escasez, relevancia, durabilidad, transferibilidad, imitabilidad, sustituibilidad, complementariedad y apropiabilidad.

En todo caso, para que sean valiosos, el acceso a los recursos y capacidades no debe ser fácil para cualquier empresa, ya que si fuese así dejarían de generar ventajas competitivas, perdiendo todo su valor diferencial. Además, deben estar relacionados con alguno de los factores clave de éxito en la industria donde compite la empresa, de forma tal que la ventaja competitiva que se pueda obtener se mantenga en el tiempo y no sea de fácil acceso a los competidores (por medio de la imitación o la adquisición del recurso o incluso la capacidad ${ }^{5}$ ). Las preferencias empresariales se adscribirán a aquellos recursos o capacidades de nivel superior, que poseen una elevada ambigüedad causal o gozan de la ventaja de mover primero. A esto hemos de añadir que, en la medida que no existan alternativas a éstos, mayor será la seguridad de la que gozará nuestra empresa.
La valoración de los recursos y capacidades poseídos por una empresa, así como la determinación de su escasez y relevancia, nos permitirán identificar las potencialidades de la misma para la consecución de ventajas competitivas. Dichas ventajas han de mantenerse durante el tiempo necesario para generar beneficios, han de ser duraderas e inimitables -o al menos difíciles de imitar, valiéndose para ello de las imperfecciones de la información y, en la medida de lo posible, de su especificidad-. En caso de que el competidor opte por su adquisición, el desarrollo de los recursos y las capacidades debe ser tal que, la relación generada entre el stock y el flujo, permita el aprovechamiento privativo de su potencial dentro de la empresa y su entorno específico. Por último, debemos considerar que el objetivo final no debe ser otro que la apropiación de las rentas generadas por dichos recursos. Este es el futuro de la empresa y se asegura en base al desarrollo, mantenimiento y extensión de estos recursos y capacidades, ajustándolos a las necesidades que le van surgiendo a la organización.

Activos intangibles y recursos humanos como elementos originarios de recursos como la cultura empresarial, la acumulación de conocimientos, el nombre comercial o la reputación son, entre otros, los que determinan la ventaja competitiva, permitiendo que las empresas obtengan una mejor posición competitiva

5 Esta autora inglesa ya comenzó a definir la teoría del crecimiento, basándose en los recursos intangibles, en 1959. Consideró el crecimiento desde el punto de vista de la oferta, que también es base para la definición de la Teoría de Recursos y Capacidades. 
y afectando positivamente a su capacidad productiva (Penrose, 1995) ${ }^{6}$. Podemos decir que los recursos y capacidades, derivados mayoritariamente de los recursos humanos que la empresa posee, son capaces de crear verdaderas ventajas competitivas a largo plazo. En resumidas cuentas, la inversión que se realice en intangibles y en recursos humanos, debe ser un objetivo prioritario empresarial a todos los niveles, partiendo de la dirección general, que debe valorar la necesidad de estos recursos, para posteriormente desarrollar con éxito las actuaciones empresariales, considerando que a largo plazo se puedan obtener mayores beneficios económicos y financieros. Estos recursos poseerán una clara importancia estratégica, basada en las características de: heterogeneidad, no fungibilidad, opacidad, inmovilidad funcional, acumulabilidad, y generación de externalidades y sinergias. Su utilización en otros productos, o incluso en otros sectores con una contribución constante, los hace poseedores de un gran valor, que en su mayoría deriva de una naturaleza basada en el conocimiento.

Las intervenciones asociadas a los recursos y capacidades que poseen las empresas son, en su mayoría, resultado de las actuaciones de los recursos humanos. La empresa necesita generar nuevos productos o servicios y, para ello, precisa personal que pueda desarrollar este objetivo en base a un proyecto estratégico preestablecido por la misma, siguiendo una serie de pautas organizativas. En resumidas cuentas las cuatro competencias básicas distintivas serán: estratégicas, tecnológicas, organizativas y personales (Ansoff, 1965). Las tres primeras, relacionadas con los componentes interno y externo de los intangibles y la última, con las competencias de los recursos humanos.

\section{Análisis Empírico de los modelos explicativos de la competitividad}

A continuación proponemos dos modelos explicativos de la competitividad de las empresas acuícolas en España, con ellos tratamos de contrastar la validez y aplicabilidad de la teoría objeto de exposición al comienzo de este artículo. Así trataremos de colegir la prevalencia de factores internos relacionados con la teoría de recursos y un factor exógeno vinculado a la presencia en mercados exteriores (propensión exportadora) ${ }^{7}$.

6 Al ser el único factor de carácter exógeno que hemos utilizado para explicar la competitividad, no hemos creído conveniente definir los fundamentos teóricos que lo podrían explicar, esto es, obviamos el análisis crítico de la competitividad según las teorías que explican el comercio internacional como las clásicas, neoclásicas y modernas, que hacen especial hincapié en los rendimientos crecientes de escala y el aumento de las escalas productivas.

7 De modo indirecto podemos corroborar la relación (por otra parte, recurrentemente referida en la literatura) entre competitividad y exportaciones (en este caso, a través del referente al logaritmo de la facturación). 
Competitividad de la acuicultura española: Modelos explicativos

Cruz González, María M.; Guisado Tato, Manuel y Sánchez Sellero, Francisco J.

\section{Cuadro 1. Ficha Técnica de la Investigación Empírica}

\begin{tabular}{|c|c|}
\hline Universo & $\begin{array}{l}\text { Sector Acuícola desde un perspectiva amplia -bases } \\
\text { de datos OESA, JACUMAR, SABI, ARDÁN, PESCA2 } \\
\text { Y ACUI2006-: } \\
\text { - Empresas productoras. } \\
\text { - Proveedores. } \\
\text { - Empresas de comercialización. }\end{array}$ \\
\hline Ámbito Geográfico & Nacional \\
\hline Método de acceso a la información & $\begin{array}{l}\text { Cuestionario postal } \\
\text { (apoyado por fax y correo electrónico) }\end{array}$ \\
\hline $\begin{array}{l}\text { Censo o población objetivo de la } \\
\text { investigación }\end{array}$ & 748 empresas \\
\hline Tamaño de la muestra & 99 empresas (índice de respuesta: $13,2 \%$ ) \\
\hline Perfil del encuestado & $\begin{array}{l}\text { Director Técnico, Gerente } \\
\text { o Departamento de Calidad }\end{array}$ \\
\hline Error muestral & $\pm 9,2 \%$ \\
\hline Nivel de confianza & $95 \%(z=1,96)$ \\
\hline Período del trabajo de campo & Octubre 2006 a Mayo 2007 \\
\hline
\end{tabular}

Fuente: Elaboración Propia.

\subsection{Metodología y datos}

Hemos realizado un análisis empírico que comprende información primaria (procedente de encuestas directas) y secundaria (derivada de la base de datos $\mathrm{SABI}$ ). El trabajo de campo derivó como resultado un tamaño muestral de 99 empresas acuícolas que dieron respuesta a todos los ítems planteados (de las cuales 75 son productores, 13 proveedores y 11 empresas de comercialización). Como se especifica en la ficha técnica adjunta, la investigación presenta un error muestral de $\pm 9,2 \%$, para un nivel de confianza del $95 \%(z=1,96)$, suponiendo la estimación más desfavorable de las proporciones $p$ y $q(p=q=0,5)$ y teniendo en cuenta un factor de corrección finita de 0,9321 (que relaciona tamaño poblacional y muestral).
La información procedente de los cuestionarios relativa a las 99 empresas comprendidas dentro del universo poblacional, se complementó con siete ratios financieros de cada una de ellas con origen en la base de datos SABI para el período 20062007 (Central de Balances del Banco de España y Registro Mercantil) (Cuadro 1).

Hemos incorporado los siguientes ratios como significativos de la situación económico-financiera de la empresa: el promedio de ingresos de explotación en los tres últimos años (log tamaño), la rentabilidad económica promedio en dicho período (rtabeca), el coste laboral anual en promedio por empleado (costelab), el porcentaje promedio del capítulo de gastos de personal sobre volumen de ventas (Gastos Personal), el valor añadido en porcentaje respecto a los ingresos de explotación (Valor Añadido), el número de 
trabajadores del ejercicio 2007 ( $n^{\circ}$ trab) y la cifra promedio de productividad en los últimos tres ejercicios cerrados (prodvd). Como variables explicativas cuyos datos fueron obtenidos de los cuestionarios obtuvimos resultados para la temporalidad de la contratación (Temporalidad), el porcentaje promedio del volumen de actividades subcontratadas respecto a la facturación de la empresa (subcontrata), el porcentaje promedio de gasto privado en formación y en investigación e innovación sobre el nivel de ventas (Gasto en Formación, PorcGtolDI), el porcentaje promedio de investigadores respecto al total de la plantilla de la empresa (Porclnvest), el número de patentes utilizadas, ya sean generadas o adquiridas (Patentes Totales) y el promedio de propensión exportadora (export).

Posteriormente dicha información, fruto de los orígenes comentados, dio lugar a la generación de una base de datos en el programa SPSS 15.00, a partir de la cual se procedió al estudio descriptivo y contraste de hipótesis individuales $y$ agregadas a través del instrumental estadístico del propio programa y el procedente del paquete estadístico AMOS 7.0 para la realización del análisis de ecuaciones estructurales.

El perfil del encuestado al que hemos solicitado información sobre el establecimiento empresarial; así dada la naturaleza global, genérica y en algunos casos específica de la información solicitada hemos considerado adecuado enviar nuestra carta de presentación al Director General o Gerente de la empresa, aunque también hemos aceptado en algunos casos las valoraciones del Director Técnico o de Calidad en función de las activida- des desarrolladas por la empresa y las funciones desempeñadas por aquél. Hemos escogido este perfil por el hecho de que las políticas de innovación, formación, desarrollo tecnológico, vigilancia sectorial y relaciones con terceros suelen tener un fuerte componente estratégico y su responsabilidad recae generalmente en los directivos seleccionados.

\subsection{Modelos, variables e hipótesis}

En cuanto al análisis conducente a la determinación de factores explicativos de la competitividad empresarial, hemos creído pertinente proponer dos modelos para su contraste. El primero de ellos de carácter factorial, que hemos complementado con ciertas relaciones entre indicadores, para comprobar incidencias directas e indirectas respecto a la competitividad. Para finalmente acabar con un análisis path de relaciones puras entre variables observables. Así de este modo definimos dos hipótesis para su posterior contraste.

Hipótesis 1: "La competitividad como variable latente se relaciona positiva y significativamente con la rentabilidad, la productividad, la propensión exportadora, el tamaño empresarial, el valor añadido, el coste laboral, la posesión de patentes y mayores niveles de gasto e inversión en I+D+i".

Hipótesis 2: "La productividad se relaciona positiva y significativamente con la rentabilidad económica, el valor añadido, la especialización y la posesión de patentes, al tiempo que empresas acuícolas que presentan un mayor número de empleados realizan mayores esfuerzos en formación, investigación y gestión del conocimiento, presentando 
Competitividad de la acuicultura española: Modelos explicativos

Cruz González, María M.; Guisado Tato, Manuel y Sánchez Sellero, Francisco J.

un mayor coste laboral, propensión exportadora y un menor porcentaje de gastos de personal respecto a empresas acuícolas con un menor número de trabajadores".

Para la verificación y confirmación de la primera hipótesis proponemos un modelo factorial explicativo (Figura 2) de la competitividad de las empresas acuícolas españolas en el que hemos utilizado lo 7 ratios o cocientes provenientes de la base de datos SABI y las 7 valoraciones subjetivas del directivo de cada empresa, mencionadas anteriormente.

Para la verificación y confirmación de la segunda hipótesis realizamos un análisis path, que aplicaremos a continuación en el segundo modelo de la investiga- ción (Figura 3), refiere relaciones directas e indirectas entre los principales ratios o cocientes de trascendencia empresarial en tanto a su competitividad. Retomamos las 14 variables observables o indicadores del modelo factorial o de medida previo ( 7 ratios SABI y 7 valoraciones subjetivas del directivo acuícola) eliminando la referencia a variable latente alguna; como era el caso de la competitividad.

\section{Resultados de la investigación}

En función de la primera hipótesis planteada y, tras la necesarias tareas de reespecificación del modelo exploratorio (Figura 4), contrastamos la incidencia po-

Figura 2. Modelo general de factores de competitividad exploratorio

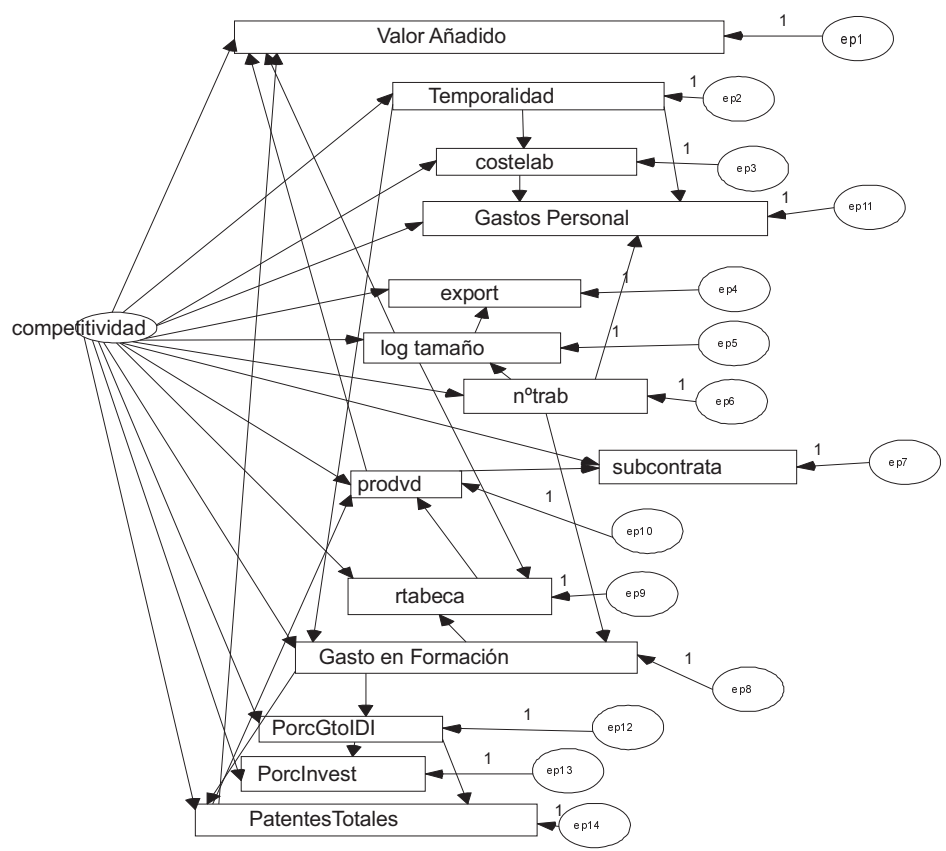

Fuente: Elaboración Propia. 


\section{Figura 3. Modelo sobre competitividad path exploratorio}

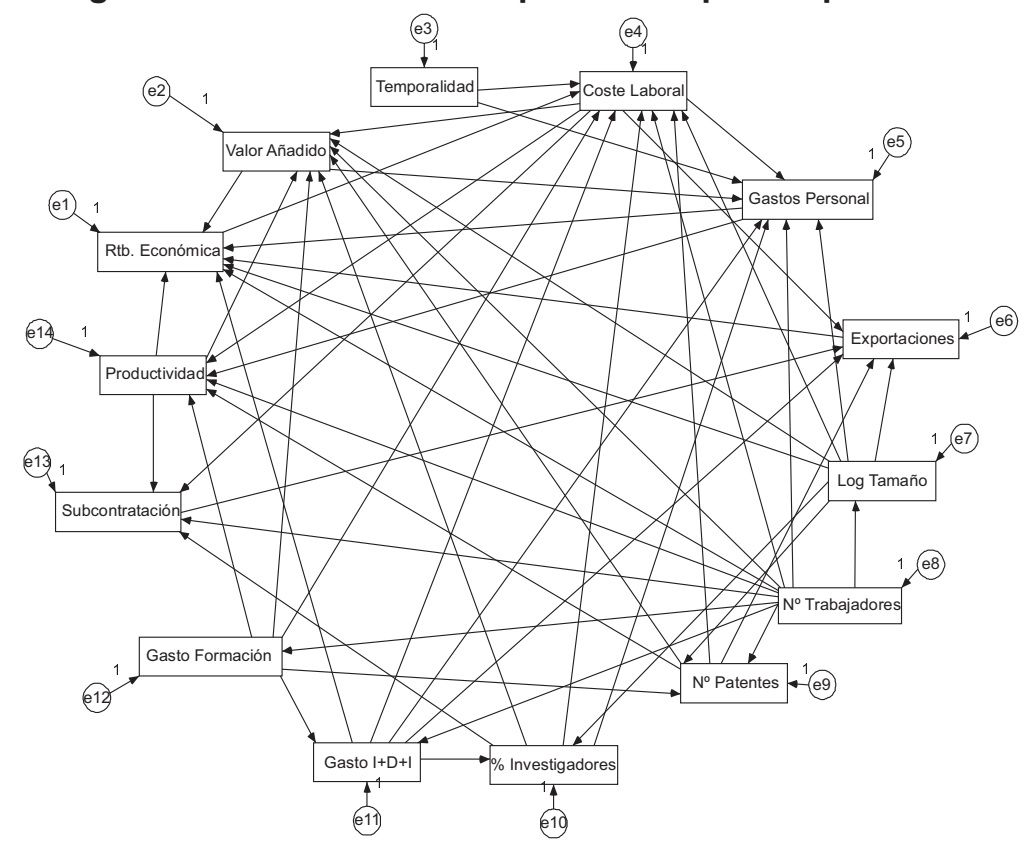

Fuente: Elaboración Propia.

sitiva sobre el tamaño (entendido como facturación o ingresos de explotación, así como con el número de trabajadores), sobre la rentabilidad económica, sobre la posesión de patentes, la productividad (en este caso de modo indirecto, a través de las patentes totales) y la propensión exportadora $^{8}$, siendo negativa su incidencia sobre los niveles de gasto e inversión en I+D+I, en principio de forma indirecta a través del porcentaje de investigadores sobre plantilla (esta constatación contrainductiva se debe a que la investigación en acuicultura se realiza básicamente a través de institutos públicos y centros tecnológicos, en los cuales los niveles de rentabilidad o productividad empresarial o de la institución son poco relevantes o anecdóticos, siendo la investigación la actividad propia o "función social", a ello se une el hecho de que la iniciativa investigadora de los grandes grupos acuícolas en España les lleve a crear divisiones o empresas específicas con personalidad jurídica independiente dentro del grupo, lo qureduce su incidencia sobre la renta-

8 El efecto sinérgico es superior al complementario, si hablamos a nivel estratégico, aunque también es más difícil de conseguir. La adición de ambos efectos, nos lleva a definir el efecto cartera, que establece cómo utilizar y acumular los recursos con eficiencia y eficacia (Itami, 1987). 
Competitividad de la acuicultura española: Modelos explicativos

Cruz González, María M.; Guisado Tato, Manuel y Sánchez Sellero, Francisco J.

Figura 4. Modelo general factores competitividad confirmatorio

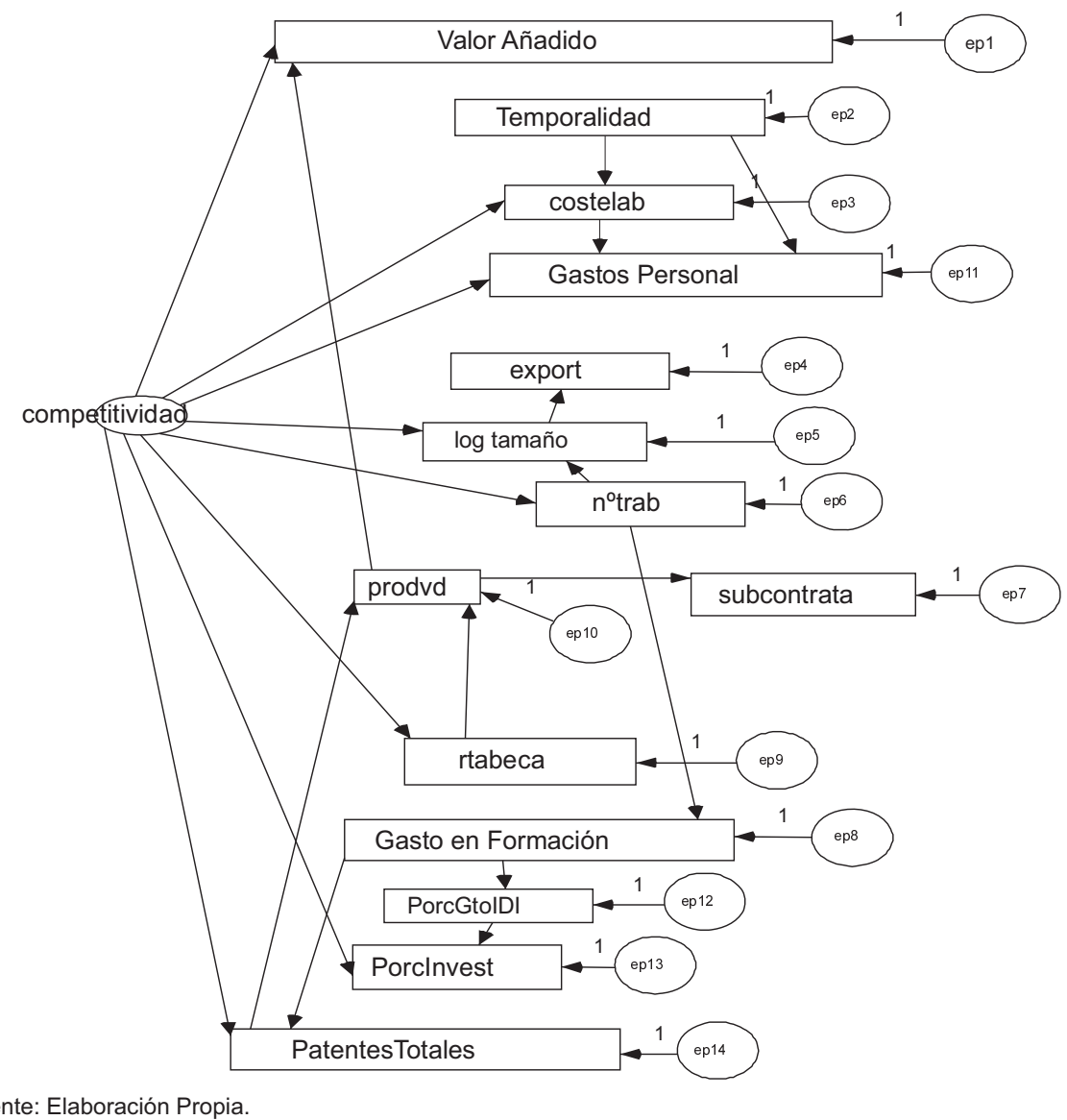

bilidad, la productividad o el tamaño; a partir de ambos factores que alteran o contaminan la incidencia positiva y natural de la investigación sobre la competitividad, podemos explicar cabalmente la constatación del modelo confirmatorio que parece chocar con la razón).

Resulta también, hasta cierto punto paradójico que la competitividad se relacione negativamente con el porcentaje de valor añadido; si bien numerosos autores que defienden procesos de integración en sistemas productivos locales y especialización flexible se apresurarían a argumentar que la competitividad se relaciona directamente con la especialización; por lo tanto, serán más competitivas aquellas empresas que se especializan en una o muy limitadas actividades frente a aquellas otras que integran muchas actividades y tareas (globalmente su valor añadido es mayor, pero son menos eficientes en la utilización de recursos respecto a las primeras). 
De modo accesorio a la hipótesis, contrastamos la incidencia negativa del abuso de la contratación temporal (fórmula de contratación creciente entre las empresas, pero cuya utilización masiva es propia de empresas que confunden objetivos, que guarda vinculación inversa con el coste laboral; el cual sí se relaciona positiva y significativamente con la competitividad y la productividad). Con ello no queremos decir que las empresas deban descuidar el coste laboral como elemento competitivo en términos de coste, sino que hemos comprobado las vinculaciones positivas y estadísticamente significativas entre competitividad, coste laboral, especialización, productividad y rentabilidad económica (la explicación sería la siguiente: "si una empresa presenta un coste laboral promedio superior a otra, esta circunstancia será el resultado de una mayor cualificación, especialización y productividad de sus trabajadores, elemento que se complementa con una mayor propensión exportadora de dichas empresas", circunstancia que el modelo path refrenda estadísticamente mediante los indicadores pertinentes).

En este punto, observamos como a pesar de pregonarse la importancia del coste laboral y la amenaza irrefrenable de procesos de deslocalización en acuicultura, las empresas más rentables, con un mayor nivel de productividad, rentabilidad y presencia en mercados exteriores presentan mayores costes laborales (este hecho de pagar comparativamente mejor a sus empleados no es incompatible con la incidencia negativa sobre la competitividad que hemos comprobado en el porcentaje de gastos de personal sobre gastos totales -en este punto entran en juego las subvenciones e incentivos públicos a la actividad acuícola que generan ineficiencia y competencia desleal globalmente, por regiones y cultivos-).

Finalmente citar múltiples relaciones evidenciadas en el modelo, como son las relaciones positivas entre el número de trabajadores y el gasto privado en formación, dicha partida y la posesión de patentes, el número de trabajadores y la facturación, el número de patentes poseídas y la productividad de la empresa, la relación entre rentabilidad económica y productividad, el porcentaje gasto privado en formación y el porcentaje de gasto en $I+D+I$, el coste laboral y el porcentaje de gastos de personal sobre le total de los mismos en la empresa y la productividad respecto al valor añadido y el porcentaje de subcontratación de actividades (en consonancia con el criterio antes referido, de las querencias por la especialización en el sector acuícola en España).

Para la segunda hipótesis $y$, teniendo en cuenta la necesaria reespecificación del modelo, presentamos en la Tabla 1 el valor y significación de las cargas factoriales estandarizadas, pudiendo derivarse otras consecuencias propias de la especificación del sector acuícola en España, objeto de análisis. Esto es, hemos querido resaltar, con la hipótesis, las principales derivaciones de la aplicación del modelo y que redundan en un conocimiento profundo de las diversas realidades que se substancian en dicho sector de actividad.

Con el referido análisis Path, hemos comprobado una fuerte, positiva y significativa vinculación entre el ratio o cociente de productividad promedio de los tres últimos años y los niveles de ren- 
Competitividad de la acuicultura española: Modelos explicativos

Cruz González, María M.; Guisado Tato, Manuel y Sánchez Sellero, Francisco J.

Tabla 1. Valor y nivel de significación de las cargas factoriales estandarizadas

\begin{tabular}{|c|c|c|c|c|}
\hline & & & \multicolumn{2}{|c|}{ Modelo 1} \\
\hline & & & Cargas estand. & Valor $\mathbf{t}$ \\
\hline$n^{0}$ trab & $<-$ & Competitividad & 0,277 & 2,327 \\
\hline Gtoform & $<-$ & $n^{\circ}$ trab & 0,267 & 2,742 \\
\hline Rtabeca & $<-$ & Competitividad & 0,312 & 3,204 \\
\hline Patentes & $<-$ & Competitividad & 0,168 & 1,978 \\
\hline Patentes & $<-$ & Gtoform & 0,531 & 6,395 \\
\hline Logtamaño & $<-$ & $n^{\circ}$ trab & 0,44 & 6,743 \\
\hline Prodvd & $<-$ & Patentes & 0,153 & 2,034 \\
\hline Prodvd & $<-$ & Rtabeca & 0,595 & 7,517 \\
\hline Costelab & $<-$ & Temporalidad & $-0,179$ & $-2,061$ \\
\hline Costelab & $<-$ & Competitividad & 0,639 & 2,7 \\
\hline Logtamaño & $<-$ & Competitividad & 0,554 & 3,322 \\
\hline Gtoidi & $<-$ & Gtoform & 0,182 & 1,937 \\
\hline Vañadido & $<-$ & Competitividad & $-0,237$ & $-2,2$ \\
\hline Gtospersonal & $<-$ & Costelab & 0,953 & 3,011 \\
\hline Vañadido & $<-$ & Prodvd & 0,438 & 4,752 \\
\hline Export & $<-$ & Logtamaño & 0,187 & 1,985 \\
\hline Subcontrata & $<-$ & Prodvd & 0,222 & 2,257 \\
\hline Invest & $<-$ & Gtoidi & 0,747 & 11,87 \\
\hline Gtospersonal & $<-$ & Temporalidad & 0,185 & 1,924 \\
\hline Gtospersonal & $<-$ & Competitividad & $-1,485$ & $-2,432$ \\
\hline Invest & $<-$ & Competitividad & $-0,223$ & $-2,599$ \\
\hline
\end{tabular}

tabilidad económica, valor añadido y porcentaje de subcontratación (como indicador del grado de especialización de actividades en la empresa), al tiempo que se corrobora como la tenencia de un mayor número de patentes redunda en una mayor productividad de la empresa.

Respecto al tamaño empresarial medido a través del número de empleados, constatamos en el modelo path con- firmatorio (Figura 5) relaciones directas y significativas con el gasto promedio en formación, la facturación de la empresa y el valor añadido, e indirectas, a través de su incidencia sobre el logaritmo de la facturación, sobre la propensión exportadora, el coste laboral y el porcentaje de gastos de personal sobre gastos totales. Igualmente identificamos relaciones indirectas con las políticas internas de inno- 
Tabla 1. Valor y nivel de significación de las cargas factoriales estandarizadas (Continuación)

\begin{tabular}{|c|c|c|c|c|}
\hline & & & \multicolumn{2}{|c|}{ Modelo 2 (path) } \\
\hline & & & Cargas estand. & Valor $\mathbf{t}$ \\
\hline Gasto Formación & $<-$ & $\mathrm{N}^{\circ}$ Trabajadores & 0,267 & 2,742 \\
\hline Log Tamaño & $<-$ & $\mathrm{N}^{\circ}$ Trabajadores & 0,594 & 7,303 \\
\hline Gasto I+D+I & $<-$ & Gasto Formación & 0,182 & 1,837 \\
\hline$\%$ Investigadores & $<-$ & Gasto I+D+I & 0,75 & 11,702 \\
\hline $\mathrm{N}^{\circ}$ Patentes & $<-$ & Gasto Formación & 0,545 & 6,443 \\
\hline$\%$ Investigadores & $<-$ & Log Tamaño & $-0,211$ & $-3,295$ \\
\hline Productividad & $<-$ & $\mathrm{N}^{\circ}$ Patentes & 0,202 & 2,011 \\
\hline Valor Añadido & $<-$ & Log Tamaño & $-0,541$ & $-4,571$ \\
\hline Valor Añadido & $<-$ & $\mathrm{N}^{\circ}$ Trabajadores & 0,207 & 1,965 \\
\hline Coste Laboral & $<-$ & Temporalidad & $-0,184$ & $-2,234$ \\
\hline Coste Laboral & $<-$ & $\mathrm{N}^{\circ}$ Patentes & 0,274 & 3,306 \\
\hline Valor Añadido & $<-$ & $\%$ Investigadores & $-0,188$ & $-2,174$ \\
\hline Gastos Personal & $<-$ & Log Tamaño & $-0,565$ & $-6,397$ \\
\hline Coste Laboral & $<-$ & Log Tamaño & 0,384 & 4,56 \\
\hline Gastos Personal & $<-$ & $\%$ Investigadores & 0,227 & 2,986 \\
\hline Exportaciones & $<-$ & Log Tamaño & 0,187 & 1,885 \\
\hline Subcontratación & $<-$ & Productividad & 0,208 & 2,445 \\
\hline Subcontratación & $<-$ & $\%$ Investigadores & 0,514 & 6,14 \\
\hline Subcontratación & $<-$ & Coste Laboral & 0,196 & 2,32 \\
\hline Rtb. Económica & $<-$ & Productividad & 0,622 & 7,623 \\
\hline Gastos Personal & $<-$ & Coste Laboral & 0,169 & 1,851 \\
\hline Coste Laboral & $<-$ & Rtb. Económica & 0,215 & 2,434 \\
\hline Valor Añadido & $<-$ & Coste Laboral & 0,363 & 3,799 \\
\hline Valor Añadido & $<-$ & Productividad & 0,534 & 5,044 \\
\hline Gastos Personal & $<-$ & Valor Añadido & 0,447 & 4,545 \\
\hline Productividad & $<-$ & Gastos Personal & $-0,499$ & $-3,95$ \\
\hline
\end{tabular}

vación, incidencia negativa a través del logaritmo de la facturación (circunstancia ya comentada en el modelo factorial previo) y positiva si nos fijamos en la relación con los gastos en formación, sea como fuere, el tamaño empresarial se relaciona con la gestión del conocimiento y la innovación. 
Competitividad de la acuicultura española: Modelos explicativos

Cruz González, María M.; Guisado Tato, Manuel y Sánchez Sellero, Francisco J.

\section{Figura 5. Modelo sobre competitividad path confirmatorio}

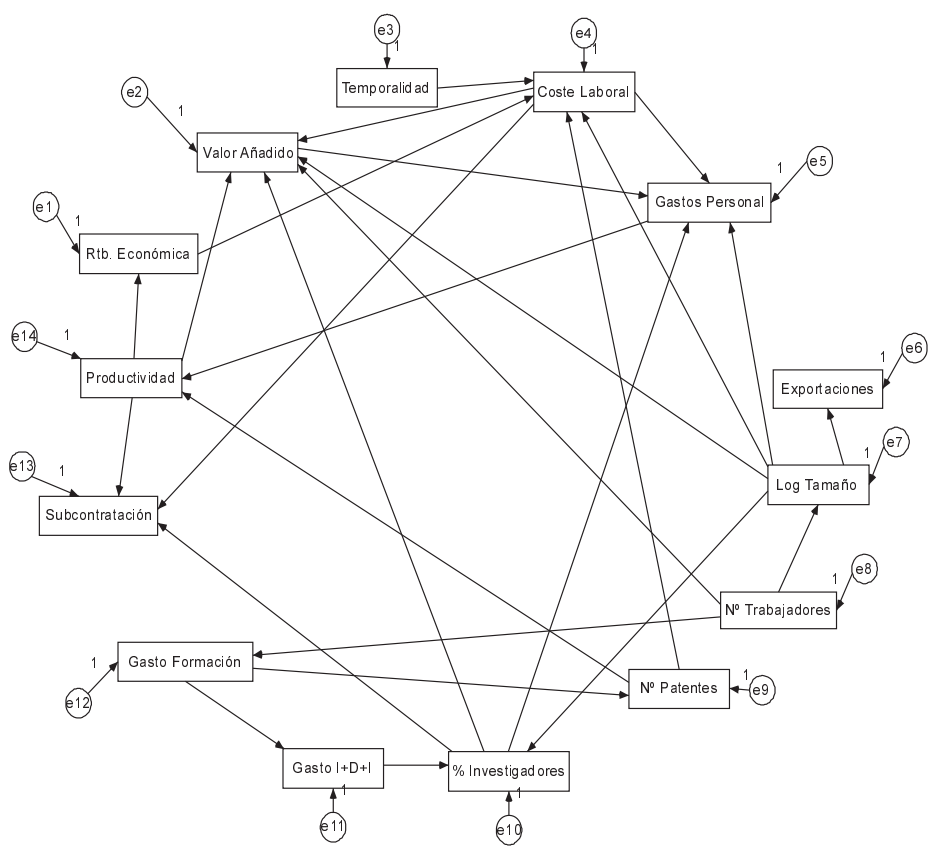

Fuente: Elaboracion propia.

Adicionalmente constatamos en el modelo path confirmatorio, otras 14 relaciones entre variables no directamente explicitadas en la hipótesis 2. Así el modelo propuesto, que pretende contrastar los fundamentos teóricos con la evidencia empírica del sector acuícola en España, relaciona positivamente el gasto en I+D+I con el porcentaje de investigadores sobre plantilla y éste con los gastos de personal y especialización de actividades (menor valor añadido y mayor porcentaje de subcontratación, coincidente por ambas vías), el gasto en formación respecto de la posesión de patentes (y éstas con el coste laboral y la productividad).

Hallamos significación estadística suficiente para relacionar positivamente el coste laboral con el valor añadido, la rentabilidad económica, la subcontratación de actividades y el porcentaje de gastos de personal sobre gastos totales, mientras la relación es inversa respecto a la temporalidad de la plantilla (racionalmente un mayor número de empleados temporales supone la asunción de menores costes laborales). Las relaciones de indicadores o variables observables son más confusas respecto al valor añadido, así éste se relaciona positivamente con los gastos de personal, siendo las incidencias que recibe de diferentes naturalezas y significaciones. En particular, una mayor plantilla genera un mayor valor añadido, siendo inversa la relación respecto a la facturación de la empresa (esto 
es, las grandes empresas acuícolas parecen incorporar mayores niveles de especialización, circunstancia especialmente destacable en proveedores), por otra parte, un mayor porcentaje de investigadores sobre plantilla genera un menor valor añadido (elemento ya comentado en el modelo factorial de competitividad al respecto de la segregación de la innovación en grandes grupos empresariales y la existencia de centros públicos de investigación, que ahora se refuerza con la consideración de la especialización derivada de las especificidades de cada investigación). Finalmente el modelo refiere una incidencia significativa y negativa entre el porcentaje de gastos de personal sobre la productividad de la empresa (no cabe duda que la productividad se relaciona con la intensidad del capital, de la innovación, de la tecnología y no con plantillas sobredimensionadas).

En la Tabla 2 presentamos los diferentes indicadores de la bondad del ajuste de los modelos explicativos de la com- petitividad empresarial, el primero factorial y el segundo causal, sobre ellos se identifican medidas absolutas, incrementales e índices de parsimonia. Ambas modelos son aceptables, si bien el segundo destaca especialmente por su utilidad práctica (probabilidad explicada del $73,4 \%$ ) que se adiciona al buen ajuste estadístico y teórico que comparten ambos modelos.

\section{Conclusiones}

Nuestra propuesta de modelización de la competitividad de la acuicultura en España ha sido desarrollada a través de un modelo factorial y otro path, con ellos hemos tratado de contrastar los fundamentos teóricos de dicha competitividad mediante factores endógenos. De este modo, la competitividad empresarial se relaciona positiva y significativamente con el tamaño, la rentabilidad económica, la posesión de patentes, la productividad (en este caso de modo indirecto, a través

Tabla 2. Índices de bondad del ajuste de los modelos

\begin{tabular}{lcc}
\hline Medidas de la bondad del ajuste & Modelo $\mathbf{1}$ & Modelo 2 \\
\hline Chi-Cuadrado & 131,321 & 57,495 \\
Grados de Libertad & 70 & 65 \\
Chi-Cuadrado/grados libertad & 1,876 & 0,885 \\
p-value & 0 & 0,734 \\
GFI & 0,858 & 0,929 \\
AGFI & 0,787 & 0,886 \\
CFI & 0,848 & 1 \\
TLI & 0,802 & 1,026 \\
RMSEA & 0,095 & 0 \\
Hoelter 0,01 & 115 & 171 \\
\hline
\end{tabular}

Fuente: Elaboración propia. 
Competitividad de la acuicultura española: Modelos explicativos

Cruz González, María M.; Guisado Tato, Manuel y Sánchez Sellero, Francisco J.

de las patentes totales) y la propensión exportadora, siendo negativa su incidencia sobre los niveles de gasto e inversión en I+D+I. Es destacable la, aparentemente paradójica, relación inversa obtenida entre competitividad y porcentaje de valor añadido sobre facturación; en este punto podemos concluir que la competitividad en acuicultura está íntimamente relacionada o vinculada al grado de especialización de las empresas y territorios. Subrayamos finalmente que dicha competitividad se relaciona positivamente con el coste laboral, la reducción de la temporalidad y el gasto privado en formación (así concluimos una mayor competitividad en empresas que soportan un mayor coste laboral y explotan en menor medida la infinidad de posibilidades de flexibilización y temporalidad que la legislación laboral vigente habilita). Habida cuenta de estas relaciones o asociaciones entre coste laboral, temporalidad, productividad, rentabilidad económica, propensión exportadora, tamaño y competitividad, deben valorarse los fenómenos "supuestamente extrapolables" de deslocalización masiva de actividades buscando la aritmética reducción de costes laborales.

El análisis path identifica 26 relaciones significativas entre los 14 factores observables o indicadores propuestos, que contrapesan la objetividad de los ratios SABI con las aportaciones de los directivos acuícolas. Así destacamos en el modelo la relación de la productividad con la rentabilidad, el valor añadido , la especialización y la posesión de patentes (compendio de teoría de recursos y capacidades y teoría de la innovación), así como las vinculaciones entre tamaño, formación, investigación, gestión del cono- cimiento, coste laboral, propensión exportadora y menores gastos de personal (enlace entre teoría de la competitividad según ventajas de las naciones, recursos y capacidades, gestión del conocimiento e innovación).

\section{Referencias Bibliográficas}

Amit, R.; Schoemaker, P. (1993). "Strategic asset and organizational rents”, Strategic Management Journal, vol. 14, pp. 33-46.

Ansoff, I. (1965). Corporate Strategy, Mc Graw Hill, New York.

Barney, J.B. (1991). "Firms resources and sustained competitive advantage", Journal of Management, vol. 17, $\mathrm{n}^{\circ} 1, \mathrm{pp}$. 99-120.

Barney, J.B. (1997). Gaining and sustaining competitive advantage, Addison Wesley, Ohio.

Boyatzis, R.E. (1982). El administrador competente, Wiley, Nueva York.

Cañibano, L.; García-Ayuso, M. y Sánchez, P. (1999). "La Relevancia de los Intangibles para la Valoración y la Gestión de Empresas", Revista Española de Financiación y Contabilidad, Extraordinario, $n^{\circ} 100$, pp. 17-88.

Chiavenato, Idalberto (2002). Gestión del talento humano, McGraw Hill, Bogotá, Colombia.

Fernández Sánchez, E.; Montes Peón, J.M. y Vázquez Ordás, C.J. (1999). "Los Recursos Intangibles como Factores de Competitividad de la Empresa", Revista de Dirección, Organización y Administración de Empresas, $n^{\circ} 20$, pp. 83-95.

González Laxe, F. (coordinador), (2001). Avances en el desarrollo de la acuicultura marina. Instituto de Estudios Económicos, Fundación Pedro Barrié de la Maza. A Coruña. 
Grant, R. (1996). Dirección Estratégica: Concepto, técnicas y aplicaciones. Civitas, Madrid.

Grant, R. (1991). "The Resource-Based Theory of Comeptitive Advantage: implication for strategy formulation", California Management Review, vol. 33, n³, pp. 114-135.

Guerras, L.A.; Navas, J.E. (2008). La Dirección Estratégica de la Empresa: Teorías y Aplicaciones. Civitas, Madrid.

Hamel, G. y Prahalad, C.K. (1990). "The Core Competence of the Corporation", Harvard Business Review, vol.68, $n^{\circ} 3$, pp. 79-91.

Hamel, G. y Prahalad, C.K. (1995). Compitiendo por el Futuro, Ariel S.A., Barcelona.

Itami, H.; Roehlt, T. (1987). Mobilizing Invisible Assets. Harvard University Press, Cambridge MA.

Loasby, B.J. (1999). "La importancia de la teoría de Penrose para el Desarrollo de la Economía" Contribuciones a la Economía Política, Oxford University Press, vol. 18 (0), pp. 31-45.

Morcillo Ortega, P. (1997). Dirección Estrategica de la Tecnología y la Innovación: un enfoque de competencias, Civitas, Madrid.

Penrose, E. (1995). The Theory of the Growth of the Firm, Basil Blackwell, Oxford.
Polanco, E.; Fernández Polanco, J.; Ruesga, S.M. (2000). La Acuicultura: regulación, biología, fomento, nuevas tendencias y estrategia comercial. Tomo I: Análisis del desarrollo de los cultivos: medio, agua y especies. Fundación Alfonso Martín Escudero. Madrid.

Polanco, E.; Fernández Polanco, J.; Ruesga, S.M. (2000). La Acuicultura: regulación, biología, fomento, nuevas tendencias y estrategia comercial. Tomo II: Economía y gestión de la acuicultura. Fundación Alfonso Martín Escudero. Madrid.

Richardson, H.W. (1977). Regional Growth Theory, The Macmillan Press Limited, Pittsburgh.

Selznick, K.E. (1957). Leadership in Administration. Harper \& Row, New York.

Sveiby, K.E. (1997a). "The intangible assets monitor", Journal of Human Resource Costing and Accounting, vol.2, n¹, pp. 73-97.

Sveiby, K.E. (1997b). Capital Intelectual: la nueva riqueza de las empresas. Como medir y gestionar activos intangibles para crear valor. Gestión 2000, Barcelona.

Wernerfelt, B. (1984). "A Resource-Based View of the Firm", Strategic Management Journal, vol.5, pp. 171-180. 